\title{
Active Mode Remote Infrared Spectroscopy Detection of TNT and PETN on Aluminum Substrates
}

\author{
John R. Castro-Suarez, ${ }^{1,2}$ Leonardo C. Pacheco-Londoño, ${ }^{1,3}$ Joaquín Aparicio-Bolaño, ${ }^{4}$ and \\ Samuel P. Hernández-Rivera ${ }^{1}$ \\ ${ }^{1}$ ALERT DHS Center of Excellence for Explosives Research, Department of Chemistry, University of Puerto Rico-Mayagüez, \\ Mayagüez, PR 00681, USA \\ ${ }^{2}$ Molecular Spectroscopy Research Group, Antonio de Arevalo Technological Foundation, TECNAR, Cartagena, Colombia \\ ${ }^{3}$ Environmental Engineering Program, Vice-Rectory for Research, Universidad ECCI, Bogota, Colombia \\ ${ }^{4}$ Department of Physics, University of Puerto Rico, Ponce, PR 00732, USA
}

Correspondence should be addressed to John R. Castro-Suarez; johncastrosuarez@gmail.com and Samuel P. Hernández-Rivera; samuel.hernandez3@upr.edu

Received 17 October 2016; Revised 4 January 2017; Accepted 17 January 2017; Published 21 March 2017

Academic Editor: Christoph Krafft

Copyright (c) 2017 John R. Castro-Suarez et al. This is an open access article distributed under the Creative Commons Attribution License, which permits unrestricted use, distribution, and reproduction in any medium, provided the original work is properly cited.

\begin{abstract}
Two standoff detection systems were assembled using an infrared telescope coupled to a Fourier transform infrared spectrometer, a cryocooled mercury-cadmium telluride detector, and a telescope-coupled midinfrared excitation source. Samples of the highly energetic materials (HEMs) 2,4,6-trinitrotoluene (TNT) and pentaerythritol tetranitrate (PETN) were deposited on aluminum plates and detected at several source-target distances by carrying out remote infrared spectroscopy (RIRS) measurements on the aluminum substrates in active mode. The samples tested were placed at $1-30 \mathrm{~m}$ for the RIRS detection experiments. The effect of the angle of incidence/collection of the IR beams on the vibrational band intensities and the signal-to-noise ratios $(S / N)$ were investigated. Experiments were performed at ambient temperature. Surface concentrations from 50 to $400 \mu \mathrm{g} / \mathrm{cm}^{2}$ were studied. Partial least squares regression analysis was applied to the spectra obtained. Overall, RIRS detection in active mode was useful for quantifying the HEMs deposited on the aluminum plates with a high confidence level up to the target-collector distances of $1-25 \mathrm{~m}$.
\end{abstract}

\section{Introduction}

The detection and identification of highly energetic materials (HEMs), commonly called explosives, and related devices are an important priority for security and counterterrorism applications [1-4]. Defense and security agencies continuously support research and development strategies for the development of efficient sensing systems that help detect HEM. When used in public places, such as airports, stadiums, maritime, and railway or coach stations, these systems can help prevent or minimize damage that could be caused by terrorist attacks [4].

Investigations on the development of sensors involving analytical methodologies that enable faster, more sensitive, less expensive, and simpler determinations to facilitate the trace identification of explosives in different fields of interest for national defense have increased in recent years [5]. Modern detection systems are routinely used to prevent these events. These are based on ionization techniques accompanied by separation schemes, pyrolysis, gas phase reactions, interaction with radiation, color tests, immunochemical reactions between HEMs and their specific antibodies, and so forth. These techniques have proven to be useful for explosive detection in different phases (solid, liquid, and gas) on various substrates or complex matrixes (such as soil, air, and water) [5-10]. However, in most cases, they require some type of sample preparation for subsequent chemical analysis.

Since each chemical substance has its own distinctive fingerprint spectrum, vibrational techniques such as Raman 
spectroscopy (RS) and Fourier transform infrared spectroscopy (FT-IRS) exploit this advantage over other analytical techniques and make them optimal for the identification of a large range of high explosives, precursors of homemade explosives, and related compounds. Among the advantages that these vibrational spectroscopy techniques offer are the possibility of analyzing samples with different chemical compositions (organic and inorganic), minimum or no sample preparation, and minute explosive particles which can be readily analyzed. These techniques have been used to characterize, detect, quantify, and discriminate HEMs, biological and chemical agents (or their simulants), toxic industrial compounds, and other threat substances [12-16]. These spectroscopic techniques have the advantage that they can be used remotely in spectral detection mode and hyperspectral imaging mode [4-8]. Remote detection is the operational capability in which the instrumentation and operator remain separated from the sample by some distances (range) while measuring some properties of the target [17]. In remote infrared spectroscopy (RIRS), vibrational signatures can be obtained at distances a few tens of meters to several tens of meters between the target and the observer. This detection modality provides a way of performing real-time analysis, in which no sample preparation or operator contact is required. Rapid cycle times are typical, and enough chemical information on the target HEMs can be obtained to identify, quantify, and discriminate signals from the matrix support or other interfering substances. These capabilities make RIRS a useful technique for sensing for HEMs, and they further prevent or minimize the possibility of harm caused by terrorist action in cases where energetic material is set off [2]. Other techniques available for remote detection of HEM include Surface Enhanced Raman Scattering (SERS), Remote Raman Spectroscopy (RRS), Laser-Induced Breakdown Spectroscopy (LIBS), and Tunable Diode Laser Absorption Spectroscopy (TDLAS). SERS is not feasible for remote detection at long ranges and requires sample preparation. LIBS in comparison with RIRS has a lower selectivity because RIRS can provide vibrational information which is unique for each substance. TDLAS is limited to gases, and moreover, it suffers from the same limitation as LIBS. In comparison with RIRS and RRS, RRS can only analyze particles in remote detection mode. RIRS is amenable to layers, particles, or traces. A comparison between the detection limits is not possible because units of measurement are different: for the molecular layers that can be analyzed by RIRS, this is in mass/area, and for particles, it is the total mass analyzed [11]. If the explosive exists on the surface as a thin layer, the backscattering signal is low. When the explosive was present on the surface as discrete particles (crystals), the backscattered RIRS signal is significantly improved.

RIRS detection is the most versatile of the remote spectroscopy-based technologies because it can measure the presence of many chemicals deposited on substrates at near-trace to trace levels at a distance [18, 19]. PachecoLondoño et al. [11] built an active RIRS detection system by coupling a bench FT-IR interferometer to a gold mirror and detector assembly for the detection of trace amounts of TNT and RDX explosives on reflective surfaces in the range of $1.0-3.7 \mathrm{~m}$. Suter et al. studied the spectral and angular dependence of scattered midinfrared light from surfaces coated with explosive residues (TNT, RDX, and tetryl) detected at a $2 \mathrm{~m}$ remote distance [20]. An external cavity quantum cascade laser (QCL) provided tunable excitation between 1250 and $1428 \mathrm{~cm}^{-1}$ [19]. Kumar et al. measured the diffuse reflection spectrum of solid samples such as explosives (TNT, RDX, and PETN), fertilizers (ammonium nitrate and urea), and paints (automotive and military grade) at a distance of $5 \mathrm{~m}$ using a midinfrared supercontinuum light source with a $3.9 \mathrm{~W}$ average output power [21].

In this report, RIRS detection experiments were performed using an open-path FT-IR interferometer. Experiments were carried out in active mode using a telescope-coupled MIR source. The effect of the source-detector-target angle on the IR spectra of PETN was evaluated. Using this sensing modality, partial least squares (PLS) regression calibrations were obtained, and the root mean square errors of crossvalidations (RMSECV) and coefficients of determination $\left(R^{2}\right)$ were used as criteria to judge the quality of the detection methodologies.

\section{Materials and Methods}

2.1. Reagents. The reagents used in this investigation included HEMs and solvents. 2,4,6-Trinitrotoluene (TNT) was acquired from Chem Service, Inc. (West Chester, PA, USA) as a crystalline solid (99\%, min. purity; 30\% water content). PETN was synthesized and purified in the laboratory according to the methods described by Ledgard [22]. Methanol (99.9\%, HPLC grade), dichloromethane $\left(\mathrm{CH}_{2} \mathrm{Cl}_{2}, \mathrm{HPLC}\right.$ grade), and acetone (99.5\%, GC grade) were purchased from Sigma-Aldrich Chemical Co. (Milwaukee, WI, USA) and were used to deposit the HEM samples at various surface concentrations onto aluminum $(\mathrm{Al})$ plates used as substrates.

2.2. Sample Preparation. Sample preparation is an important process in the validation of remote detection experiments of analytes present as trace residues on the substrates. A sample smearing technique was used to deposit HEM samples on metal substrates [15]. Al plates with $1.0 \mathrm{ft} . \times 1.0 \mathrm{ft}$. $\left(929 \mathrm{~cm}^{2}\right)$ dimensions were used as a sample support for the HEM targets. Acetone was used to clean the Al substrates. After cleaning, the plates were allowed to dry before depositing the desired HEM target. A small amount of dichloromethane or methanol was used to dissolve the desired HEM samples to be deposited on the test substrates. Then, a Teflon stub $3 \mathrm{~cm} \times 15 \mathrm{~cm}$ was used to smear the samples on the Al plates. The amount of HEM that remained on the Teflon stub after sample smearing was negligible. The nominal surface concentrations obtained by the smearing technique used were 50,100, 200,300 , and $400 \mu \mathrm{g} / \mathrm{cm}^{2}$.

2.3. Experimental Setup. For the remote detection experiments, the HEM-coated Al plates were placed at the target positions, and the ambient temperature, the plate temperature, and the relative humidity were measured during the experiments. Next, active mode RIRS detection experiments were carried out using the two optical systems illustrated in 


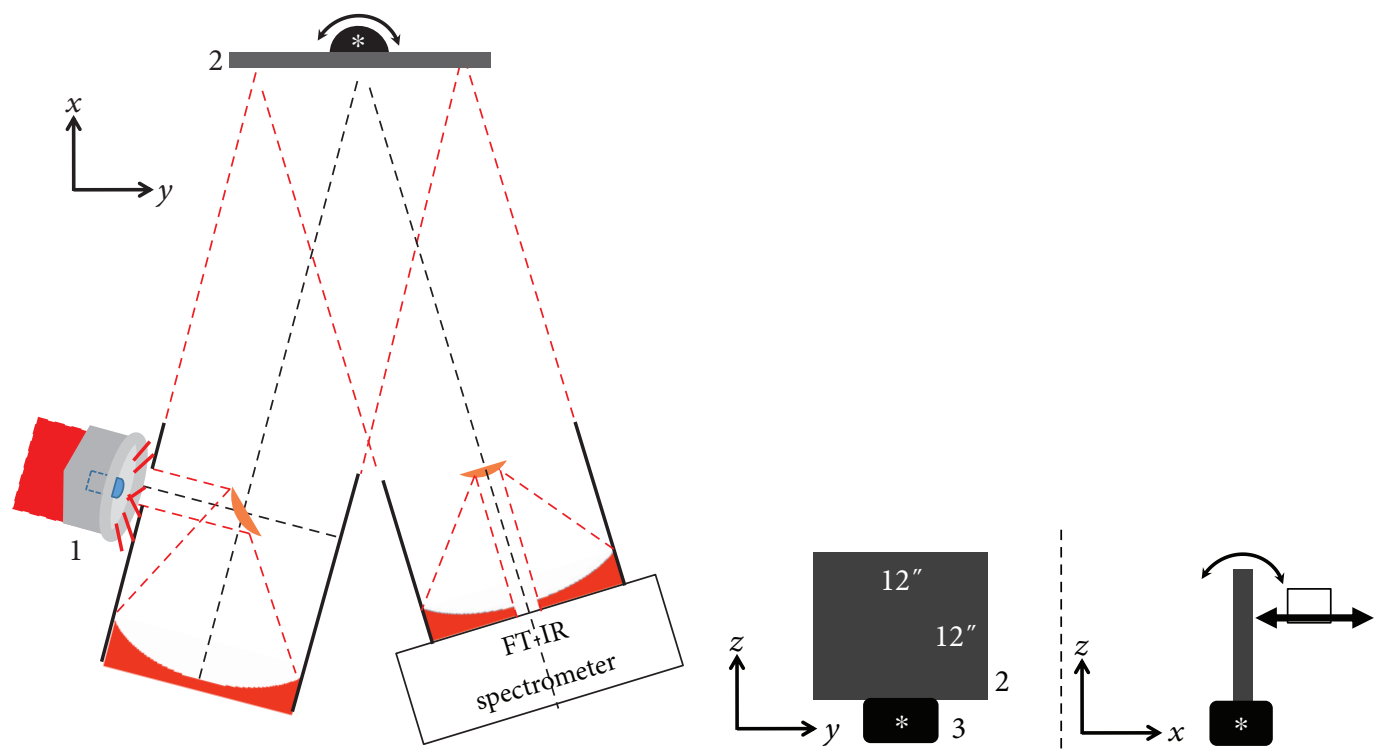

(a)

(b)

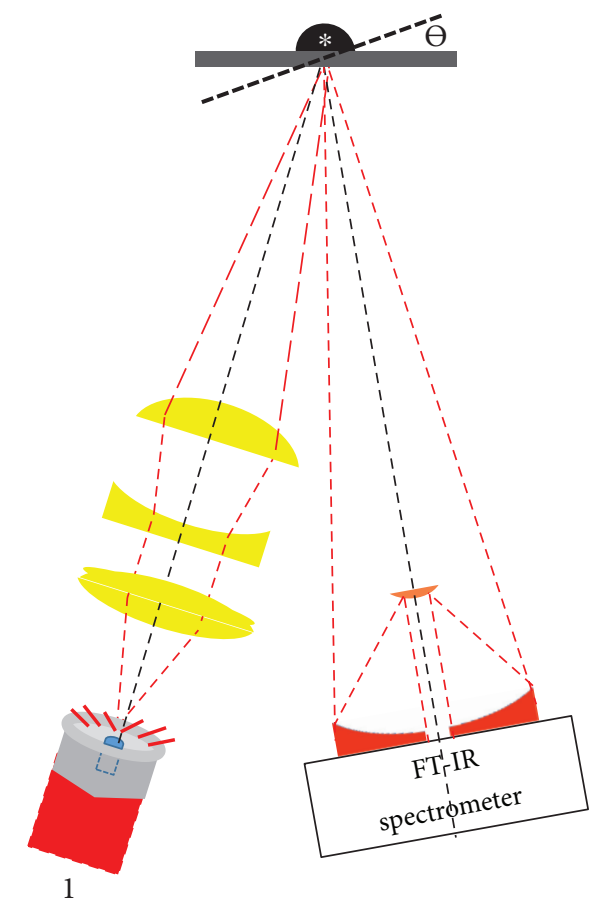

(c)

FIGURE 1: FT-IR interferometer configuration: (a) active mode setup for standoff measurements using reflective telescope: 1, IR source, 2, Al plate; (b) plate mount: 3, tilting mount; (c) active mode setup for standoff measurements using refractive telescope.

Figures 1(a) and 1(c). In the first optical system, a midinfrared (MIR) reflective telescope coupled to a heated oxide globar source (Figure 1(a)) was used. In the second optical system, a midinfrared (MIR) refractive telescope coupled to the globar source as shown in Figure 1(c) was used.

In the remote sensing experiments, the IR beam from the globar source was not modulated by the interferometer before interacting with the target and sat side-by-side with the MIR reflective collector telescope that was close coupled to the FT interferometer (Figure 1(a)). The experiments were conducted in back-reflection mode. The FT-IR spectrometer used was an open-path interferometer, model EM27 (Bruker Optics, Billerica, MA, USA). The optical bench consisted of a compact, enclosed, and desiccated Michelson-type interferometer equipped with $\mathrm{ZnSe}$ windows, an internal blackbody calibration source, and a $\mathrm{KBr}$ beam splitter. This system had a very fast native focal ratio $(f / 0.9)$ and a field of view (FOV) of $30 \mathrm{mrad}\left(1.7^{\circ}\right)$. For the optical system illustrated in Figure 1(a), the transmitter source telescope had a diameter of $6 \mathrm{in}$., a focal ratio of $f / 4$, and gold-coated mirrors with a 


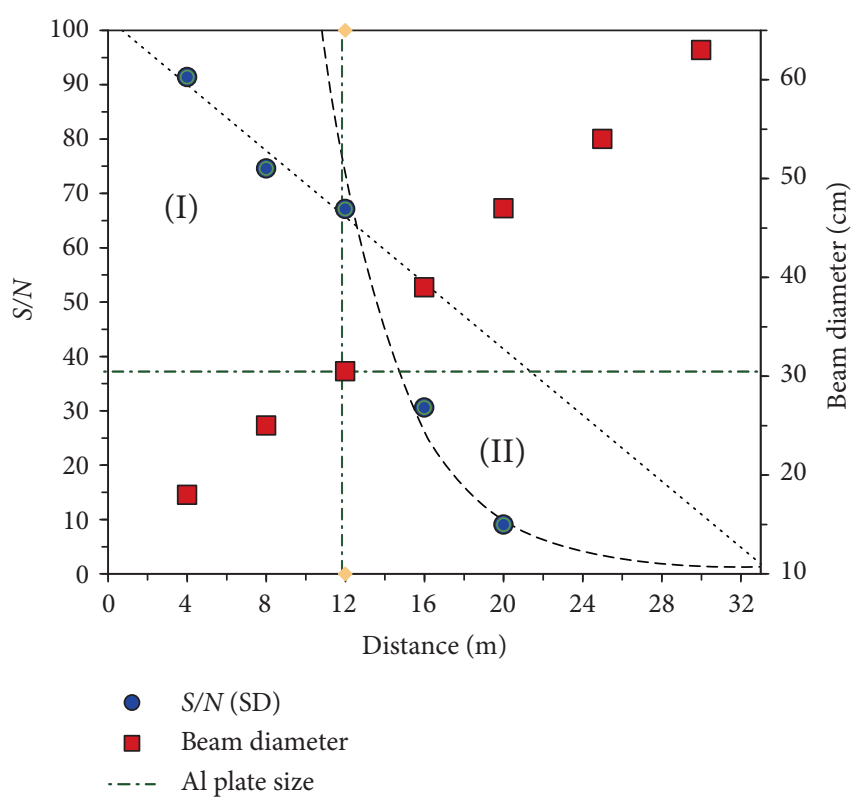

FIgURE 2: Signal-to-noise ratio (principal $y$-axis) at various distances for active mode RIRS measurements of TNT. IR beam spot size (secondary $y$-axis) versus range. Noise levels (as standard deviation) were measured at $830-870 \mathrm{~cm}^{-1}$ and peak heights were measured for TNT signal at $790 \mathrm{~cm}^{-1}$.

FOV $\geq 7.5 \mathrm{mrad}\left(0.43^{\circ}\right)$. The receiver telescope was also $6 \mathrm{in}$. in diameter and had an $f / 3$ focal ratio and gold-coated mirrors with a FOV of $10 \mathrm{mrad}\left(0.57^{\circ}\right)$.

In the second optical system used (Figure 1(c)), the transmitter source telescope consisted of a set of three 4 in. diameter $\mathrm{ZnSe}$ lenses of $100 \mathrm{~mm}$ focal length $(f)$ for the biconvex lens, $-1000 \mathrm{~mm} f$ for the plano-concave lens, and $1000 \mathrm{~mm}$ $f$ for the plano-convex lens. The receiver telescope was the same as in the first optical layout.

In these experimental setups, the targets were carefully aligned with the source and the collector. To accomplish this, the metal plate was placed on a mount that allowed millimeter translations both horizontally and vertically, as illustrated in Figure 1(b). The target-collector distances studied were $1,4,8,12,16,20,25$, and $30 \mathrm{~m}$. A total of 10 spectra were taken for each sample at 20 scans/spectrum and a $4 \mathrm{~cm}^{-1}$ resolution. The spectra were recorded in the spectral range from 750 to $1400 \mathrm{~cm}^{-1}$. The experiments were performed at ambient temperature $\left(\sim 25^{\circ} \mathrm{C}\right)$.

IR signals were detected using an MIR closed cycle (Stirling cooled) photoconductive MCT detector with $D^{*}{ }_{\max } \sim 4 \times 10^{10} \mathrm{cmHz}^{1 / 2} / \mathrm{W}$. Background spectra of the $\mathrm{Al}$ plates with no HEM deposited on them were run for every remote distance studied. Data analyses were based on statistical routines using chemometrics. Specifically, partial least squares (PLS) regression analysis was used to perform quantification studies of the surface concentrations of the HEMs at all distances studied.

\section{Results and Discussion}

Active mode standoff IR measurements of solid HEM dissolved in an appropriate solvent and smeared on Al plates at various surface concentrations were carried out. The spectra were collected in the MIR region $\left(750-1400 \mathrm{~cm}^{-1}\right)$ using the setups described.

3.1. RIRS Detection of HEMs. Signal-to-noise ratios $(S / N)$ were calculated and plotted against the remote detection distance for active mode spectral measurements of TNT. The results for surface concentrations of $400 \mu \mathrm{g} / \mathrm{cm}^{2}$ deposited on the Al plates that were obtained using the optical system illustrated in Figure 1(a) are shown in Figure 2. $S / N$ initially decreased linearly with distance (Figure 2, blue circles; equation: $-3.03 \pm 0.02 \mathrm{~m}^{-1} * X+$ $101.96 \pm 0.01)$. However, when the detection distance was larger than $12 \mathrm{~m}$, the signal decreased with an even steeper slope $\left(\operatorname{Exp}\left(-0.26 \pm 0.04 \mathrm{~m}^{-1} * X+7.4 \pm 0.6\right)+1\right)$ reaching a $S / N$ of $\sim 3$ (calculated by extrapolation from the exponential fitting of the equation of $S / N$ versus distance, region II) at a distance of $26 \mathrm{~m}$. The decrease in the collected signal did not allow the measurement of $S / N$ for the entire distance range planned (to $60 \mathrm{~m}$ ), making the measurements accurate up to $25 \mathrm{~m}$. The two linear decreases in the collected signal were calculated from linear fittings and are shown as black and gray dotted lines in Figure 2. The difference in slope in regions I: $4-12 \mathrm{~m}$, and II: $12-25 \mathrm{~m}$, suggested a fundamental reason for the behavior and led to the measurements of the spot size of the MIR beam at the target plane. As shown in the graph, the spot diameter is smaller than the target in region I; it is exactly equal to the target at $12 \mathrm{~m}$ and is larger than the target in region II. A linear dependence of the spot diameter with the detection distance is also shown in Figure 2. When an MIR globar source was used to accomplish the spectroscopic measurements in active mode, the peak intensities decreased as the distances increased. At distances longer than $25 \mathrm{~m}$, it was not possible to visually detect some of the TNT vibrational signatures in the spectra obtained. This result was as expected since, as shown in Figure 2, the distance required for the threshold $S / N$ of 3 is $24 \mathrm{~m}$ in active mode. Furthermore, the density of infrared radiation that is transferred to the $\mathrm{Al}$ plates from the MIR source diminishes as a function of distance, leading to a smaller number of excited molecules at the target. Therefore, the detector could not register the transflection intensities of the low-intensity vibrational modes.

The active mode MIR spectra for TNT deposited on $\mathrm{Al}$ plates at several distances and surface concentrations are shown in Figure 3(a). The experiments were performed using the setup illustrated in Figure 1(a). A reference spectrum for TNT is included for comparison purposes and for assignment of the modes of the important nitroaromatic HEM. The latter was obtained by preparing a pellet of $1 \mathrm{mg}$ of microcrystalline TNT in $100 \mathrm{mg} \mathrm{KBr}$ and measuring the absorption spectrum in the macro sample chamber of a benchtop interferometer (Bruker Optics IFS-66v). The spectrum of the solid is represented by the black trace labeled "TNT ref." Upon inspection of these spectra, it is evident that the most significant TNT vibrational signatures were detected in the remote sensor measurements. In particular, an intense vibrational band at approximately $908 \mathrm{~cm}^{-1}$ was assigned to the $-\mathrm{C}-\mathrm{N}$ stretching, a vibrational band at 


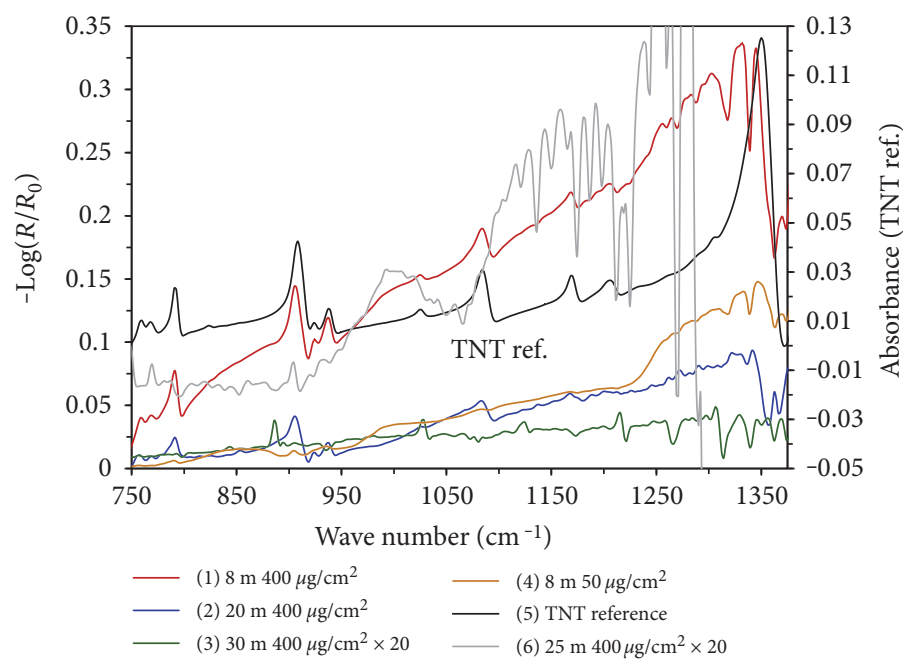

(a)

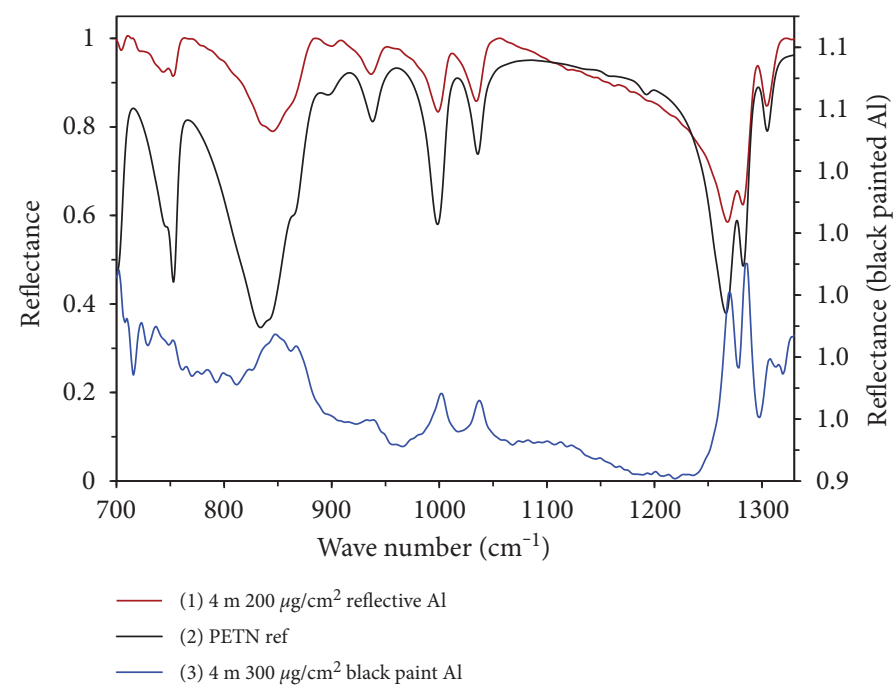

(b)

FIgURe 3: (a) Active mode RIRS spectra of TNT deposited on an Al plate measured at 8, 20, 25, and $30 \mathrm{~m}$ and surface concentrations of $400 \mu \mathrm{g} / \mathrm{cm}^{2}$ ((1), (2), (3), and (6)) and $50 \mu \mathrm{g} / \mathrm{cm}^{2}$ (4), respectively. (b) Active mode RIRS spectra of PETN deposited on Al plate and black painted $\mathrm{Al}$ at $4 \mathrm{~m}$.

$938 \mathrm{~cm}^{-1}$ was assigned to the $-\mathrm{C}-\mathrm{H}$ out-of-plane ring bending, and the symmetric stretch band of the nitro groups appeared at $1350 \mathrm{~cm}^{-1}$. All assignments were tentative. These results are all due to the conjugation of the nitro groups with the aromatic ring and agree with results from Pacheco-Londoño et al. [11], Clarkson et al. [23], and Castro-Suarez et al. [24]. These spectra were not submitted to any preprocessing routine, such as offset correction, baseline correction, smoothing, or water vapor rotational line removal. In other words, there was no common baseline for these spectra, and some spectra exhibited positive intensity ramps to higher wave number values. However, an increase in signal intensity as a function of the surface concentrations was clearly displayed without the use of chemometrics routines for distances less than or equal to $20 \mathrm{~m}$. For standoff distance of $25 \mathrm{~m}$, low-intensity TNT vibrational signals can be appreciated, which can be confirmed with the aid of chemometrics routines.

The spectral band shapes observed in remote detection mode, shown in Figure 3(a), are superimposed on a rampshaped background, and the bands themselves exhibit strong transflection band profiles. Since these measurements were performed on a reflective metal substrate, the distortion of the band profiles is expected. Similar effects have been reported in diffuse reflection infrared Fourier transform spectroscopy (DRIFT) [25] and acquired micro-IR spectra of microspheres [26]. In both cases, the distortion of the absorption line shapes is because of the reflective index that undergoes anomalous dispersion within the band. In spectroscopic experiments carried out in reflectance mode, a mixing of the absorptive and dispersive line shapes can occur, resulting in bands that have negative dips at the high wave number 
side of the peak. This will shift the maximum peak, by up to $15 \mathrm{~cm}^{-1}$, toward lower values [26]. Moreover, the mixing of absorptive and reflective line shapes can be mediated by scattering effects [26], which could also produce significant band distortions. In a paper about the line shape distortion effects in infrared spectroscopy by Miljković et al., the authors address the conditions under which the mixing of reflective and absorptive band shapes will occur. They also discuss the methods that have been developed to correct the spectral distortions [28]. Castro-Suarez et al. demonstrated that the spectra shown in Figure 3(a) have the appearance of "double pass" transmission-reflection (transflectance) spectra [24]. Transflection experiments are usually performed by placing a thin target sample on a non-IR absorbing, reflective substrate such as a polished metal surface, focusing an IR beam onto a region of interest and collecting the radiation that is reflected to the collection optics. The technique is termed transflection because most of the signal intensity collected is a transmission signal as the beam passes through the sample, reflects off the substrate, and passes through the sample again before reaching the detector [27-29].

The active mode MIR spectra for PETN deposited on $\mathrm{Al}$ plates at several distances and surface concentrations are shown in Figure 3(b), using the experimental setup illustrated in Figure 1(c). This optical system was designed to overcome the limitations of the setup illustrated in Figure 1(a). Among these was the inability to focus at a certain distance shorter than infinity, which did not allow control over the beam size at the target distance, as is shown in Figure 2. The size of the spot increased as the distance increased. A reference spectrum for PETN, obtained as previously described for TNT, is also included for reference and for facilitating band assignments. The spectra obtained contained the most significant band for identifying the HME. For PETN in Figure 3(b), important vibrational signatures appeared at $703 \mathrm{~cm}^{-1}(-\mathrm{ON}$ stretching $+-\mathrm{NO}_{2}$ rocking), $753 \mathrm{~cm}^{-1}\left(-\mathrm{ONO}_{2}\right.$ umbrella), $869 \mathrm{~cm}^{-1}$ (-ON stretching), $939 \mathrm{~cm}^{-1}$ (- $\mathrm{CH}_{2}$ torsion), $1003 \mathrm{~cm}^{-1}$ (-CO stretching), $1038 \mathrm{~cm}^{-1}$ (-NO $\mathrm{NO}_{2}$ rocking), $1272 \mathrm{~cm}^{-1}$ (- $\mathrm{ONO}_{2}$ rocking), $1285 \mathrm{~cm}^{-1}$ ( $-\mathrm{NO}_{2}$ stretching), and $1306 \mathrm{~cm}^{-1}\left(-\mathrm{NO}_{2}\right.$ rocking) [30]. These spectra were not submitted to any preprocessing routine, such as offset correction, smoothing, or water vapor rotational line removal. As for the case of TNT, there was no common baseline for these spectra, and some spectra exhibited positive intensity ramps to higher wave number values.

The remote detection of PETN deposited on pieces of black painted car door is shown in Figure 3(b) (blue trace). This spectrum had upward-looking peaks when compared with the spectrum taken from PETN on a reflective Al substrate (red trace), and the PETN reference spectrum measured in reflectance mode with a bench spectrometer (Bruker Optics IFS$66 / v$, Bruker Optics) also exhibited downward-looking peaks. Although all spectra were recorded in transflection mode, the spectral patterns observed demonstrate the effect of the nature of the substrate on the spectral profiles of the target HEM. In transflection experiments, such as the one described above, the recorded spectra are a weighted sum of the transmission and reflection characteristics of the samples and substrates. In samples deposited on highly reflective substrates, the

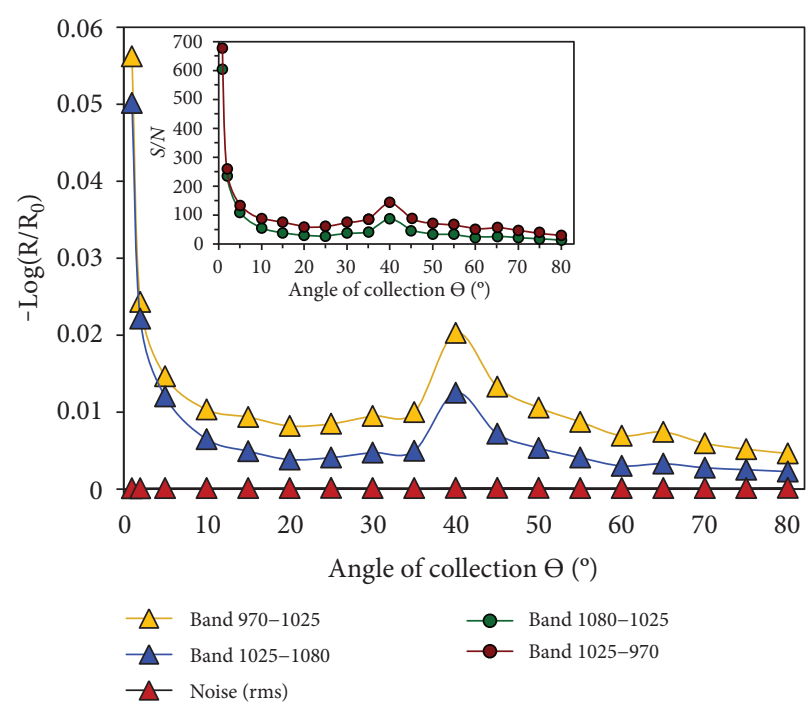

Figure 4: PETN $\left(200 \mu \mathrm{g} / \mathrm{cm}^{2}\right)$ vibrational band intensities and $S / N$ (inset) at different angles (offset) of collection $(\Theta)$.

weighting is such that the transmission signal dominates, and the reflection signal is negligible, producing spectra closely resembling those of a transmission spectrum, in this case, the PETN reference spectrum on $\mathrm{Al}$ (black spectrum). However, if the transmission signal is weak or inexistent, the reflection signal will dominate, and a reflectance spectrum is obtained, as shown by the blue trace in Figure 3(b). Castro-Suarez et al. discuss in detail the IRS spectral profile of targets deposited on nonreflective substrates [24].

In active mode RIRS detection of targets residing on metal substrates, the signal strength is highly dependent on the alignment between the IR source, the target, and the detector. Therefore, a study of the effect of the angle of collection $(\Theta)$ of the IR beam on the intensity of the vibrational signal was required. In this work, $\Theta$ represents the angular offset from the maximum signal intensity, assigned to back reflection alignment $(\Theta=0)$. The optical layout illustrated in Figure 1(c) was used for remote detection at $1 \mathrm{~m}$. The influence on the intensity and the $S / N$ for two vibrational bands of PETN/Al at a surface concentration of $200 \mu \mathrm{g} / \mathrm{cm}^{2}$ on the angle of collection is shown in Figure 4. Bands analyzed were those at $970-1025 \mathrm{~cm}^{-1}$ and at $1025-1080 \mathrm{~cm}^{-1}$. The band intensities were taken as the average height of the five spectra. The reflected intensities $(S)$ were represented as $-\log \left(R / R_{0}\right)$, which is the function proportional to the surface concentration of the analytes. The noise level $(N)$ was taken as the average root mean square (rms) values of the five spectra measured in the range of $1105 \mathrm{~cm}^{-1}$ to $1130 \mathrm{~cm}^{-1}$, that is, the standard deviation of all data points in this region [30]. $S / N$ was calculated as the ratio between the band intensities and the noise level. Intensities of the bands and the $S / N$ were calculated by varying the angle of collection from $1^{\circ}$ to $80^{\circ}$, as illustrated in Figure 4 . The intensities measured for the two bands decreased by $50 \%$ and $25 \%$ when the collection angle changed from $1^{\circ}$ to $2^{\circ}$ and from $1^{\circ}$ to $5^{\circ}$, respectively. For collection angles equal to or larger than $10^{\circ}$, the intensities decreased considerably compared 


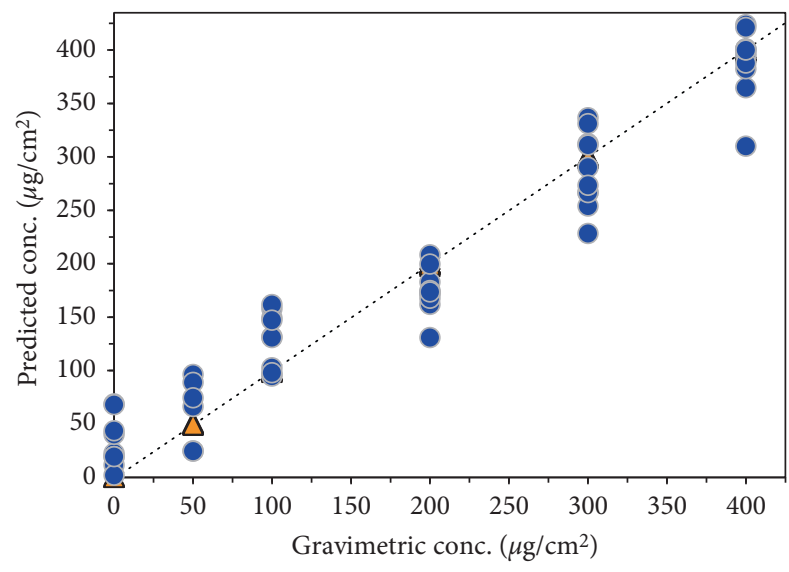

$\begin{array}{ll}\triangle & 20 \mathrm{~m} \text { TNT } \\ - & 25 \mathrm{~m} \text { TNT } \\ \cdots & y=x\end{array}$

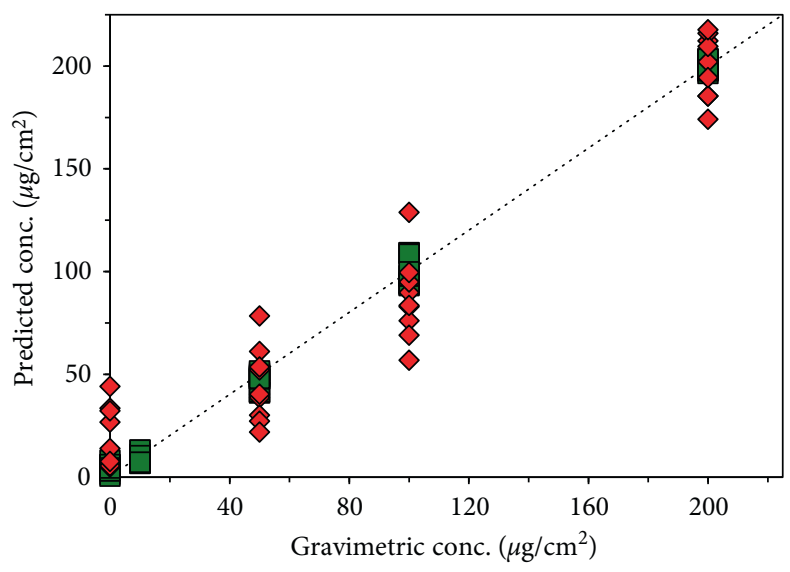

$\square \quad \operatorname{PETN} 1^{\circ}$

$\operatorname{PETN} 60^{\circ}$

(a)

Figure 5: (a) Predicted versus true surface concentration for TNT explosives on Al plates at $20 \mathrm{~m}$ and $25 \mathrm{~m}$. (b) Predicted versus true surface concentration for PETN explosives on Al plates at $1^{\circ}$ and $60^{\circ}$.

to those of $1^{\circ}$. The intensity decrease depended on the spectral band selected. For the band at $1038 \mathrm{~cm}^{-1}$, the intensity of $0.05\left(\Theta=1^{\circ}\right)$ was reduced to $\sim 0.004\left(\Theta>10^{\circ}\right)$. Thus, the intensity was reduced to $8 \%$ when compared to $\Theta=1^{\circ}$. However, for the band at $1003 \mathrm{~cm}^{-1}$, the intensity decreased from $0.056\left(\Theta=1^{\circ}\right)$ to $\sim 0.008\left(\Theta>10^{\circ}\right)$ or $14 \%$ of its original value. As shown in Figure 4 , at collection angles from $35^{\circ}$ to $50^{\circ}$, both spectral bands showed an increase in intensity. This result seems to be due to a geometrical factor. The graph of $S / N$ versus angle of collection, shown as an insert in Figure 4, has a similar behavior to the graph of intensity versus angle of collection. At small angles $\left(\Theta=2^{\circ}\right.$ to $\left.5^{\circ}\right)$, the $S / N$ decreases significantly. However, even at $\Theta=80^{\circ}$, $S / N$ is equal to fifteen, so HEM characteristic spectral bands could still be detected at such large angles of incidence.

3.2. HEM Quantification Using PLS Regression. The multivariate regression algorithm known as partial least squares1 (PLS-1) was used to find the best correlation function between the HEM spectral information and the surface concentrations [31]. The Quant2 ${ }^{\mathrm{TM}}$ software of OPUS ${ }^{\mathrm{TM}}$ v. 6.0 (Bruker Optics) was used to build the models. Calibrations were performed using PLS-1 in which only one component can be analyzed separately, instead of simultaneously analyzing multiple components, as in the PLS-2 routine of chemometrics. PLS-1 regressions were used to generate chemometrics models for all of the remote distances studied. In addition, cross-validations were made, and the root mean square errors of the cross-validations (RMSECV) and coefficient of determination $\left(R^{2}\right)$ were used as indicators of the quality of the correlations obtained at the various distances studied.

Statistical treatments for the chemometrics models based on PLS-1 regressions were performed using the complete spectral region (from 700 to $1400 \mathrm{~cm}^{-1}$ ), where the nitro symmetric stretch and aromatic ring $-\mathrm{C}-\mathrm{H}$ vibrations occur in these compounds. Ten replicate spectra were used for each tested sample. In the models generated, it was not necessary to eliminate any spectrum. The method of leave-one-out cross-validation (LOOCV) was used, where each of the " $n$ " calibration samples was left out, one at a time, and the resulting calibration models were then used to evaluate the sample, which acts as an independent validation sample and provides an independent prediction of each dependent variable. This process of leaving a sample out was repeated until all of the calibration samples had been left out.

Data preprocessing is an important step in performing chemometrics calibrations. To ensure the reproducibility of the calibration samples, ten spectra of each sample (fixed surface concentration and remote distance) were acquired. The following data preprocessing steps were tested: vector normalization, first derivative, and second derivative. However, no preprocessing of the data worked better than any other preprocessing routine or combination of preprocessing routines. Therefore, no preprocessing steps were applied for achieving the best possible values for RMSECV and $R^{2}$ in the spectral region from 700 to $1400 \mathrm{~cm}^{-1}$ other than applying mean centering of each spectral variable [30]. Overall, the results indicate that the experimental setup used had good management of the external variables, such as humidity, temperature changes, and the concentrations of the samples deposited on the Al plates.

Figure 5(a) shows the results obtained for the crossvalidations carried out for the TNT spectra measured at distances of 20 and $25 \mathrm{~m}$ in active mode (represented as orange triangles and blue circles, resp.) using the experimental setup shown in Figure 1(a). Each point of the graph represents ten spectra with a fixed surface concentration $(0,50,100,200$, 300 , or $400 \mu \mathrm{g} / \mathrm{cm}^{2}$ ). In all of the correlation charts, the predicted surface concentration values (dependent variable) versus the gravimetric surface concentration values (independent variable) for $4,8,12$, and $16 \mathrm{~m}$ were similar to the correlation chart for $20 \mathrm{~m}$ distance in Figure 5(a) (orange triangles). 
TABLE 1: PLS calibration parameters for various remote distances analyzed.

\begin{tabular}{lccc}
\hline Distance $(\mathrm{m})$ & $R^{2}$ & RMSECV & LVs \\
\hline 4 & 1 & 0.457 & 8 \\
8 & 1 & 0.434 & 7 \\
12 & 1 & 0.676 & 8 \\
16 & 1 & 0.736 & 9 \\
20 & 0.9999 & 1.08 & 9 \\
25 & 0.9367 & 34.6 & 5 \\
30 & 0.8553 & 53.5 & 9 \\
\hline
\end{tabular}

However, as the distance increased above $20 \mathrm{~m}$, some of the spectral information fell below the quantification level causing the spectra for each sample to be slightly different from each other (within experimental error) and making it difficult to predict the surface concentration precisely. The fact that the RMSECV (Figure 5(a); blue circles) is larger at standoff distances greater or equal to $25 \mathrm{~m}$ can be due to the limitations of our system, Figure 1(a), in failing to focus the IR beam to a certain size at the target. For this standoff distance $(25 \mathrm{~m})$ the size of the IR beam was $>200 \%$ larger than the size of the target (see Figure 2). Thus, the radiant intensity on the target decreased considerably. This decrease was in addition to the well-established power reduction with the inverse of the standoff distance squared. For the standoff distance of $25 \mathrm{~m}$, very low intensity vibrational signals of TNT can be appreciated, mainly those at $900 \mathrm{~cm}^{-1}$ assigned to the $-\mathrm{C}-\mathrm{N}$ stretching. Low intensity bands measured at $25 \mathrm{~m}$ standoff distance are close to $S / N \sim 3$, that is, the detection limit for the experimental setup used (Figure 1(a)) calculated by extrapolation as mentioned above. Table 1 shows the results of RMSECV and $R^{2}$ obtained in the PLS models generated.

Figure 6(a) shows the plot of the regression coefficient for the PLS model of TNT detection and quantification on $\mathrm{Al}$ plates at $8 \mathrm{~m}$. This regression coefficient spectrum in the spectral range from 750 to $1375 \mathrm{~cm}^{-1}$ contains the characteristic bands of TNT that are relevant for predicting the detection/quantification of HEM/Al. In addition, Figure 6(a) also shows the loading (latent variable (LV)) plot used in the PLS model. The LVs are the variables with the largest statistical weights corresponding to the analyte characteristic bands. LV1 for the PLS model at $8 \mathrm{~m}$ is enough to describe the principal vibrational bands of TNT. However, at a $25 \mathrm{~m}$ remote distance, the important bands of TNT are also contained in LV2 for the PLS model because for detection at longer distances, it was difficult to predict the surface concentration based on only LV1.

The values obtained for RMSECV and $R^{2}$ for 25 and $30 \mathrm{~m}$, respectively, are as expected, as already discussed, falling below the threshold of $S / N$ equal to 3 (for distances $\geq 24 \mathrm{~m}$ ). Taking into account the low values of RMSECV and the high values of $R^{2}$ obtained, it is concluded that the models are useful tools for determining the surface concentration of TNT in unknown samples at a maximum distance of $20-24 \mathrm{~m}$ with the setup used for the measurements.
TABLE 2: PLS calibration parameters for various angles of collection analyzed.

\begin{tabular}{lccc}
\hline Angle of collection $\Theta\left({ }^{\circ}\right)$ & $R^{2}$ & RMSECV & LVs \\
\hline 1 & 0.9963 & 4.26 & 7 \\
20 & 0.9628 & 14.10 & 7 \\
40 & 0.9802 & 10.30 & 7 \\
60 & 0.9237 & 20.40 & 7 \\
\hline
\end{tabular}

Figure 5(b) shows the results obtained for crossvalidations carried out for the PETN spectra measured at $1 \mathrm{~m}$ in active mode configuration for two angles of collection $(\Theta)$ : $1^{\circ}$ and $60^{\circ}$ (represented as green squares and red diamonds, resp.). The data were acquired using the experimental setup shown in Figure 1(c). Table 2 shows the results of RMSECV and $R^{2}$ obtained in the PLS models generated for $\Theta=1^{\circ}, 20^{\circ}$, $40^{\circ}$, and $60^{\circ}$. In these correlation charts, each point represents ten spectra with a fixed surface concentration $(0,10,50$, 100 , or $200 \mu \mathrm{g} / \mathrm{cm}^{2}$ ). However, as the angle of collection increased, the intensities of the vibrational bands decreased considerably and made the correlation with the surface concentration difficult.

Figure 6(b) displays the regression coefficient and loading plot (LV1) for the PLS model of PETN explosive detection and the quantification of Al plates at $1 \mathrm{~m}$ and an angle of collection of $1^{\circ}$. This regression coefficient spectrum contains the characteristic vibrational bands of PETN that are significant for predicting the detection/quantification of the HEM deposited at near-trace levels on the Al substrates. Figure 6(b) also shows the first loadings used in the PLS model; the variables with the largest statistical weights correspond to the PETN characteristic bands illustrated in Figure 3(b). The values obtained for RMSECV and $R^{2}$ for the angles of collection of $1^{\circ}, 20^{\circ}, 40^{\circ}$, and $60^{\circ}$ demonstrate that it is possible to carry out statistically meaningful RIRS detection experiments with the setups described even at high incident angles. As is shown in Table 2, when $\Theta$ is equal to $40^{\circ}$, values of RMSECV and $R^{2}$ improve, which is expected from the results shown in Figure 4. Taking into account the low values of RMSECV, the high values of $R^{2}$, and the high values of $S / N$, the models were useful for determining the surface concentration of PETN in unknown samples.

\section{Conclusions}

Two remote detection IR techniques based on an open-path FT-IR interferometer have been demonstrated and used to obtain spectral information of HEM samples deposited on Al plates. High spectral quality measurements were achieved using MIR reflective and refractive telescopes coupled to remote detection systems. Detection in active mode proved to be successful for detecting TNT vibrational signatures in the range of 4-20 m, and at $25 \mathrm{~m}$ with very slight vibrational signals, which could be confirmed with the aid of chemometrics routines. The RIRS detection system worked particularly well for distances smaller than $24 \mathrm{~m}$ due to the transmitter telescope characteristics in sensing TNT. At $24 \mathrm{~m}$, the MIR beam size was as large as the target $(30 \mathrm{~cm})$, 


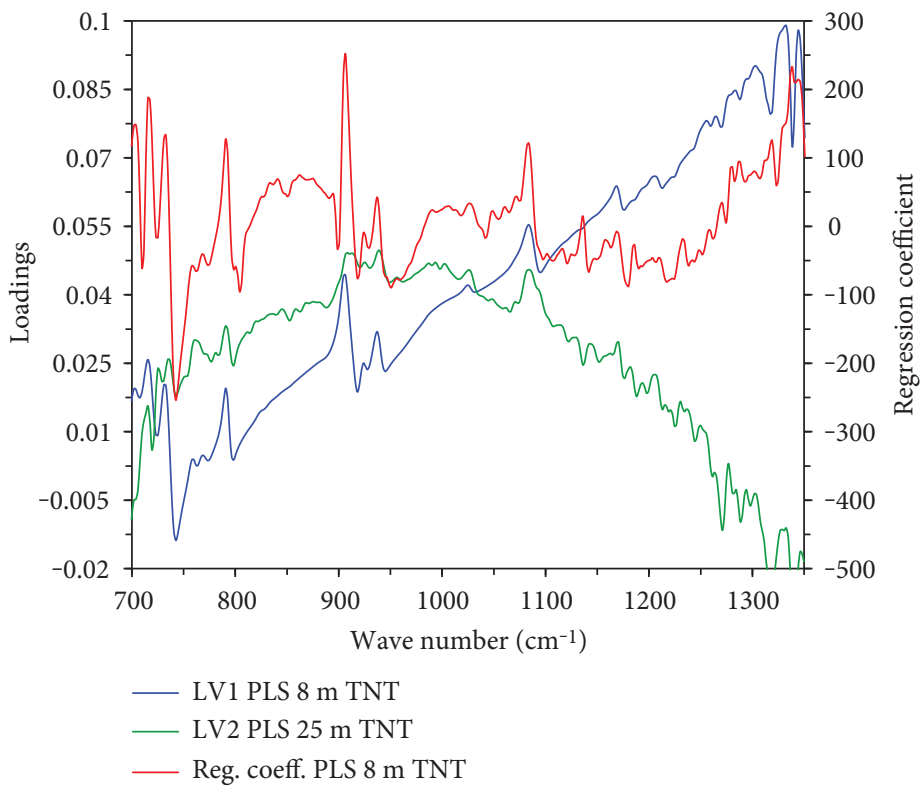

(a)

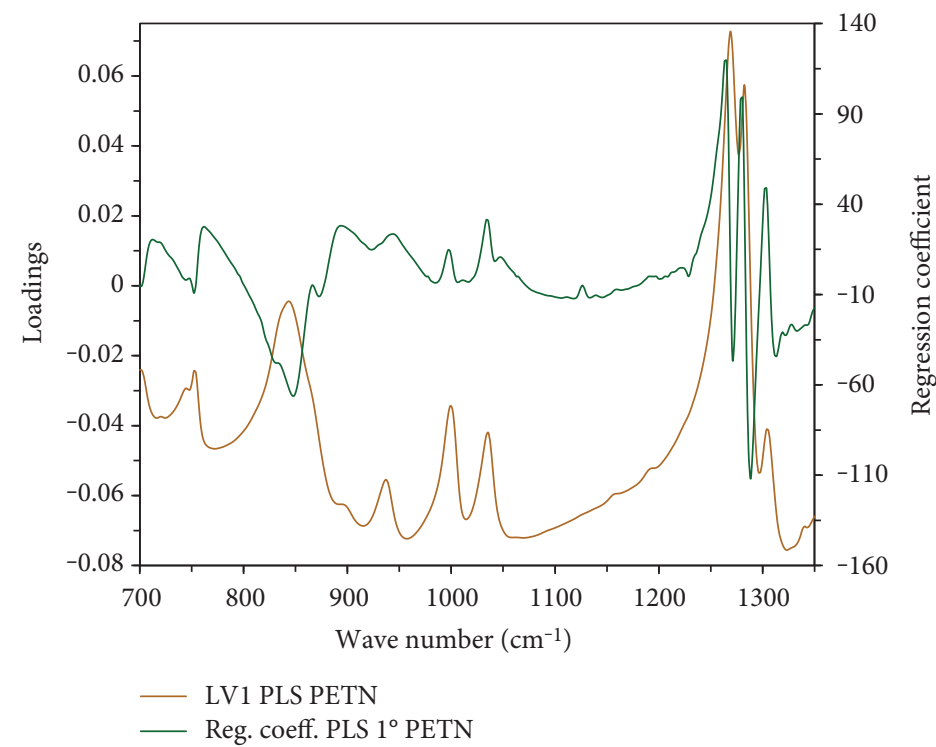

(b)

Figure 6: (a) Regression coefficient and loading plot for PLS model of detection of TNT explosives on Al plates at $8 \mathrm{~m}$ standoff distance. (b) Regression coefficient and loading plot for PLS model of detection of PETN explosives on Al plates at $1 \mathrm{~m}$ standoff distance and angle of collection $1^{\circ}$.

and the $S / N$ was 3, falling rapidly above $25 \mathrm{~m}$ using MIR reflective telescopes. However, it was necessary to align the target carefully with the detector to be able to measure with high accuracy at distances between 25 and $30 \mathrm{~m}$.

The optical system designed for detecting PETN vibrational bands in the range of $1-4 \mathrm{~m}$ by varying the angle of collection from $1^{\circ}$ to $80^{\circ}$ was equally successful. Furthermore, excellent results for RMSECV and $R^{2}$ were obtained for models generated based on PLS cross-validations for the active mode experiments.

\section{Disclosure}

The views and conclusions contained in this document are those of the authors and should not be interpreted as necessarily representing the official policies, either expressed or implied, of the U.S. Department of Homeland Security.

\section{Conflicts of Interest}

The authors report that there are no conflicts of interest. 


\section{Acknowledgments}

This material is based upon work supported by the U.S. Department of Homeland Security, Science and Technology Directorate, Office of University Programs, under Grant Award 2013-ST-061-ED0001. This contribution was also supported by the U.S. Department of Defense, Agreement no. W911NF-11-1-0152. The authors also acknowledge contributions from Dr. Richard T. Hammond from the Army Research Office, DoD.

\section{References}

[1] H. Schubert and A. Rimski-Korsakov, Stand-Off Detection of Suicide-Bombers and Mobile Subjects, Springer, DordrechtThe Netherlands, 2006.

[2] K. Banas, A. Banas, H. O. Moser et al., "Post-blast detection of traces of explosives by means of Fourier transform infrared spectroscopy," Analytical Chemistry, vol. 82, no. 7, pp. 3038-3044, 2010.

[3] M. Marshall and J. C. Oxley, Aspects of Explosives Detection, Elsevier, Oxford, 2009.

[4] M. López-López and C. García-Ruiz, "Infrared and Raman spectroscopy techniques applied to identification of explosives," TrAC Trends in Analytical Chemistry, vol. 54, no. 2, pp. 36-44, 2014.

[5] J. S. Caygill, F. Davis, and S. P. J. Higson, "Current trends in explosive detection techniques," Talanta, vol. 88, no. 1, pp. 14-29, 2012.

[6] J. Yinon, "Field detection and monitoring of explosives," TrAC Trends in Analytical Chemistry, vol. 21, no. 4, pp. 292-301, 2002.

[7] D. S. Moore, "Instrumentation for trace detection of high explosives," Review of Scientific Instruments, vol. 75, no. 8, pp. 2499-2512, 2004.

[8] D. J. Klapec and G. Czarnopys, "Analysis and detection of explosives and explosives residues review: 2010 to 2013," 17th Interpol International Forensic Science Managers Symposium, vol. 526, pp. 280-435, Lyon, France, October 8-10, 2013.

[9] A. J. Bandodkar, A. M. O'Mahony, J. Ramírez et al., "Solid-state forensic finger sensor for integrated sampling and detection of gunshot residue and explosives: towards 'lab-on-a-finger'," Analyst, vol. 138, no. 18, pp. 5288-5295, 2013.

[10] T. Caron, M. Guillemot, P. Montméat et al., "Ultra trace detection of explosives in air: development of a portable fluorescent detector," Talanta, vol. 81, no. 1, pp. 543-548, 2010.

[11] L. C. Pacheco-Londoño, W. Ortiz, O. M. Primera, and S. P. Hernández-Rivera, "Vibrational spectroscopy standoff detection of explosives," Analytical and Bioanalytical Chemistry, vol. 395, no. 2, pp. 323-335, 2009.

[12] J. L. Gottfried, "Discrimination of biological and chemical threat simulants in residue mixtures on multiple substrates," Analytical and Bioanalytical Chemistry, vol. 400, no. 10, pp. 3289-3301, 2011.

[13] W. Ortiz-Rivera, L. C. Pacheco-Londoño, J. R. Castro-Suarez, H. Felix-Rivera, and S. P. Hernandez-Rivera, "Vibrational spectroscopy standoff detection of threat chemicals," in Proceedings of SPIE 8031, Micro-and Nanotechnology Sensors, Systems, and Applications III, vol. 8031, Article ID 803129, 2011.
[14] J. Mass, A. Polo, O. Martínez et al., "Identification of explosive substances through improved signals obtained by a portable Raman spectrometer," Spectroscopy Letters, vol. 45, no. 6, pp. 413-419, 2012.

[15] J. R. Castro-Suarez, L. C. Pacheco-Londoño, M. Vélez-Reyes, M. Diem, T. J. Tague, and S. P. Hernández-Rivera, "FT-IR standoff detection of thermally excited emissions of trinitrotoluene (TNT) deposited on aluminum substrates," Applied Spectroscopy, vol. 67, no. 2, pp. 181-186, 2013.

[16] A. C. Padilla-Jiménez, W. Ortiz-Rivera, J. R. Castro-Suarez, C. Ríos-Velázquez, I. Vázquez-Ayala, and S. P. HernándezRivera, "Microorganisms Detection on Substrates Using QCL Spectroscopy, in Proceedings of SPIE: Chemical, Biological, Radiological, Nuclear, and Explosives (CBRNE) Sensing XIV, vol. 8710, Article ID 871019, 2013.

[17] J. E. Parmenter, "The challenge of standoff explosives detection," Proceedings of the 38th Annual 2004 International Carnahan Conference on Security Technology, pp. 355-358, IEEE, 2004.

[18] P. R. Griffiths, L. Shao, and A. B. Leytem, "Completely automated open-path FT-IR spectrometry," Analytical and Bioanalytical Chemistry, vol. 393, no. 1, pp. 45-50, 2009.

[19] J. R. Castro-Suarez, Y. S. Pollock, and S. P. HernandezRivera, "Explosives Detection Using Quantum Cascade Laser Spectroscopy," in Proceedings of SPIE - The International Society for Optical Engineering, Chemical, Biological, Radiological, Nuclear, and Explosives (CBRNE) Sensing XIV, vol. 8710 Article ID 871010, 2013.

[20] J. D. Suter, B. Bernacki, and M. C. Phillips, "Spectral and angular dependence of mid-infrared diffuse scattering from explosives residues for standoff detection using external cavity quantum cascade lasers," Applied Physics B, vol. 108, no. 4, pp. 965-974, 2012.

[21] M. Kumar, M. N. Islam, F. L. Terry et al., "Stand-off detection of solid targets with diffuse reflection spectroscopy using a high-power mid-infrared supercontinuum source," Applied Optics, vol. 51, no. 15, pp. 2794-2807, 2012.

[22] J. Ledgard, The Preparatory Manual of Explosives, J. Ledgard, Ed., ISBN-13: 978-0615142906.

[23] J. Clarkson, W. E. Smith, D. N. Batchelder, D. A. Smith, and A. M. Coats, "A theoretical study of the structure and vibrations of 2,4,6-trinitrotoluene," Journal of Molecular Structure, vol. 648, no. 3, pp. 203-214, 2003.

[24] J. R. Castro-Suarez, M. Hidalgo-Santiago, and S. P. HernándezRivera, "Detection of highly energetic materials on nonreflective substrates using quantum cascade laser spectroscopy," Applied Spectroscopy, vol. 69, no. 9, pp. 1023-1035, 2015.

[25] J. M. Chalmers and M. W. Mackenzie, "Some industrial applications of FT-IR diffuse reflectance spectroscopy," Applied Spectroscopy, vol. 39, no. 4, pp. 634-641, 1985.

[26] P. Bassan, H. J. Byrne, F. Bonnier, J. Lee, P. Dumas, and P. Gardner, "Resonant Mie scattering in infrared spectroscopy of biological materials-understanding the dispersion artifact," Analyst, vol. 134, no. 8, pp. 1586-1593, 2009.

[27] P. Bassan, H. J. Byrne, J. Lee et al., "Reflection contributions to the dispersion artefact in FTIR spectra of single biological cells," Analyst, vol. 134, no. 6, pp. 1171-1175, 2009.

[28] M. Miljković, B. Bird, and M. Diem, "Line shape distortion effects in infrared spectroscopy," Analyst, vol. 137, no. 17, pp. 3954-3964, 2012. 
[29] J. M. Chalmers, "Mid-infrared spectroscopy: anomalies, artifacts and common errors," in Handbook of Vibrational Spectroscopy, J. M. Chalmers and P. R. Griffiths, Eds., vol. 3, John Wiley and Sons, Chichester, 2006.

[30] P. R. Griffiths and J. A. De Haseth, Fourier Transform Infrared Spectrometry, vol. 171, John Wiley \& Sons, Hoboken, NJ, 2nd edition, 2007.

[31] W. F. Perger, J. Zhao, J. M. Winey, and Y. M. Gupta, "Firstprinciples study of pentaerythritol tetranitrate single crystals under high pressure: vibrational properties," Chemical Physics Letters, vol. 428, no. 4, pp. 394-399, 2006. 

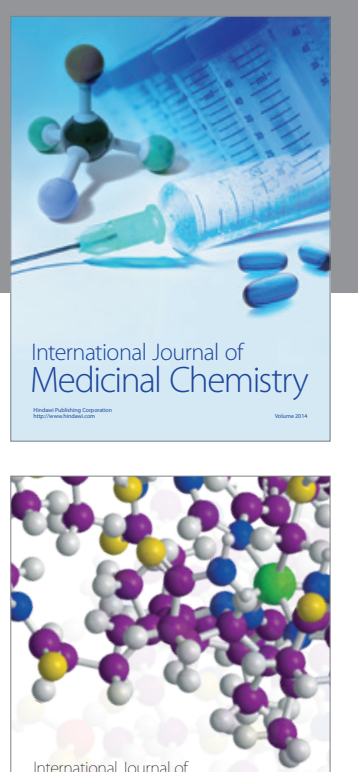

Carbohydrate Chemistry

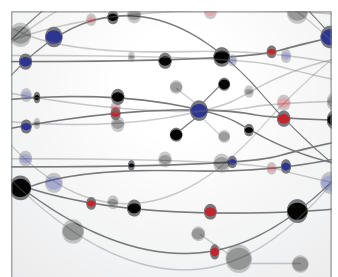

The Scientific World Journal
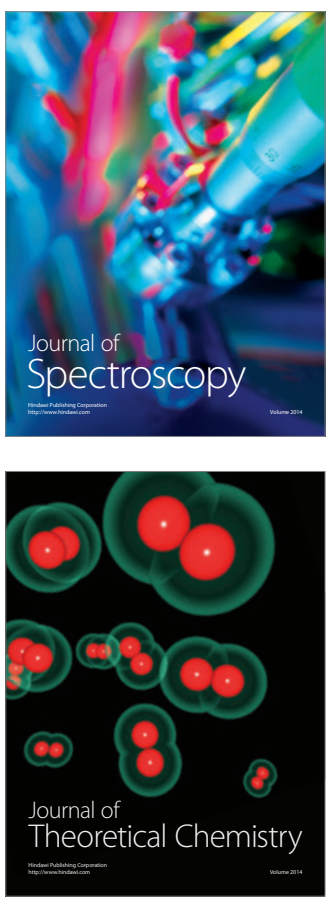
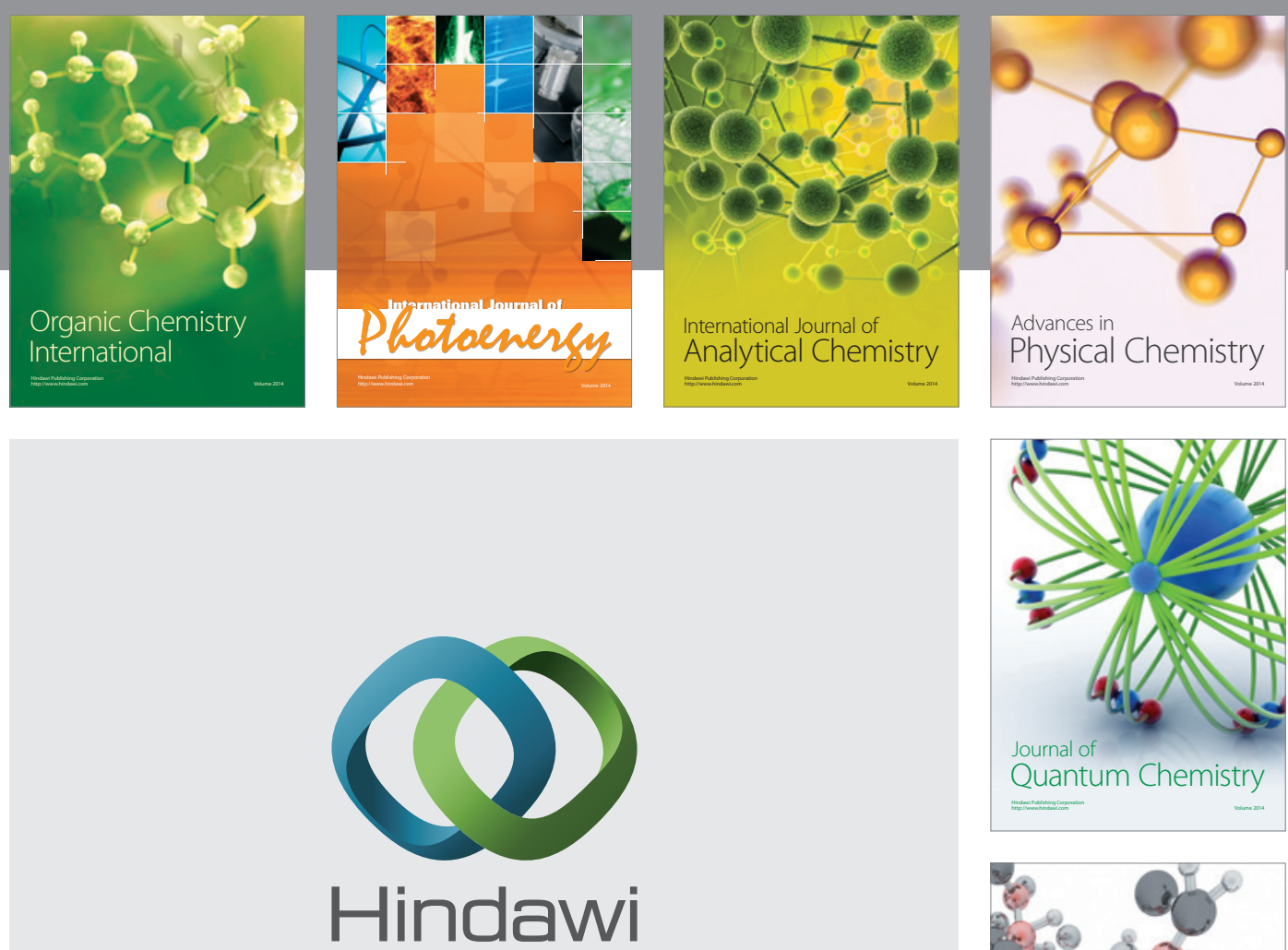

Submit your manuscripts at

https://www.hindawi.com

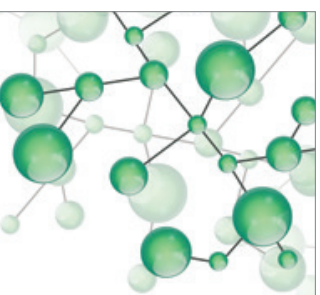

International Journal of

Inorganic Chemistry
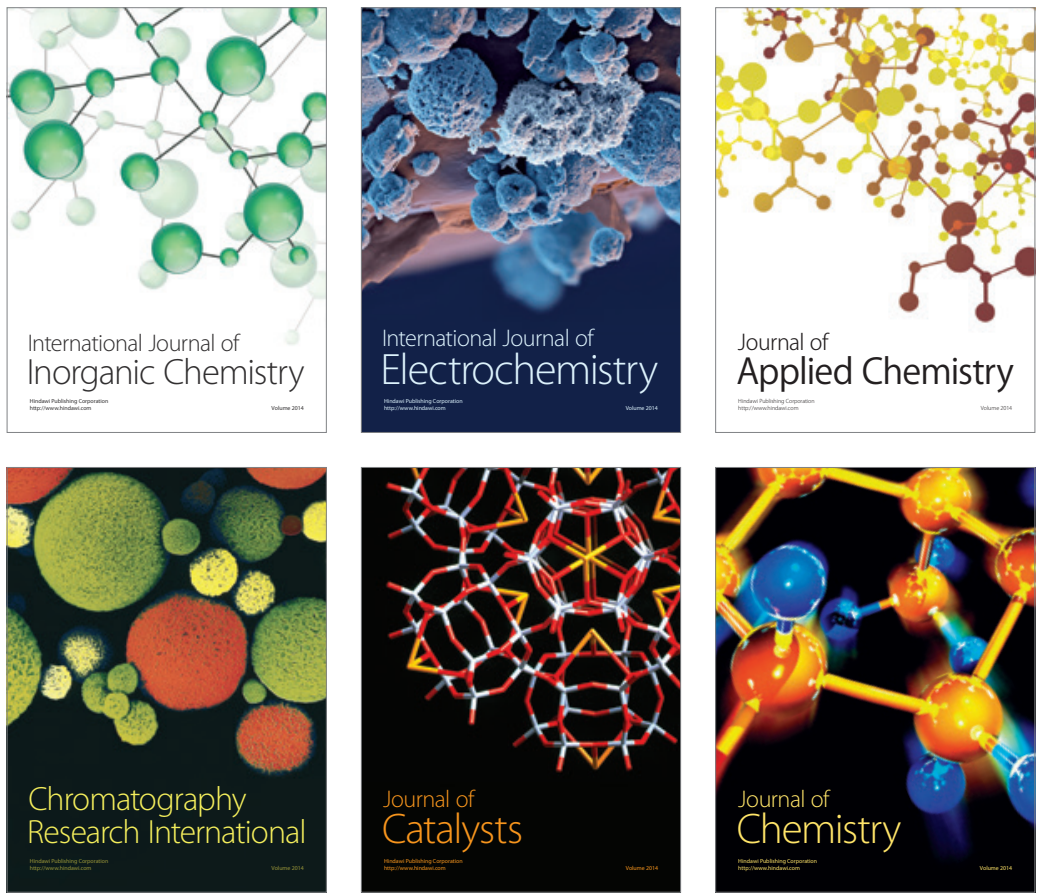

Journal of

Applied Chemistry
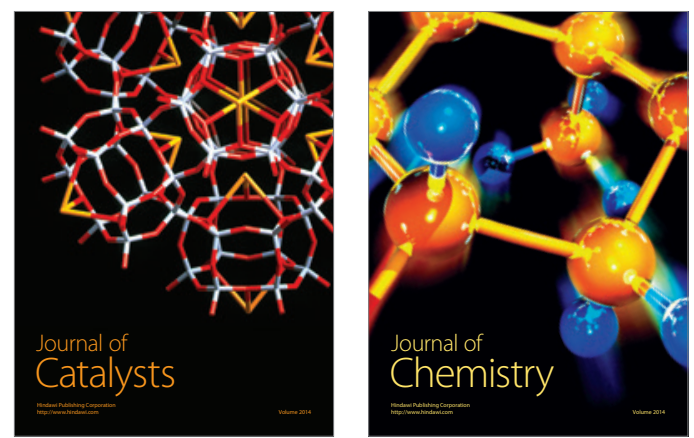
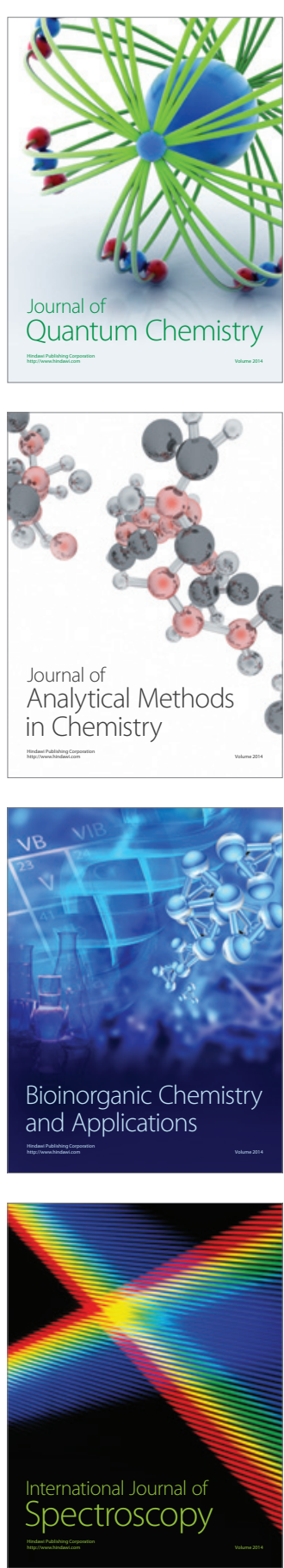